\title{
Why the Parts of Absolute Space are Immobile
}

\author{
Nick Huggett \\ Philosophy, University of Illinois at Chicago
}

February 10, 2007

\begin{abstract}
Newton's arguments for the immobility of the parts of absolute space have been used to licence several proposals concerning his metaphysics. This paper clarifies Newton. Notably, it demonstrates, contrary to Nerlich (2005), that Newton does not appeal to the identity of indiscernibles, but rather to a view about de re representation. Additionally, I show, contrary to DiSalle (1994), that the argument does not reveal Newton to be an anti-substantivalist. Its premises entail that 'Leibniz shifts' in space are impossible, but they also entail that all motion is the relative motion of bodies; hence they cannot be core doctrines.
\end{abstract}

Since Newton took absolute motion to be motion with respect to absolute space, it is not surprising that he held the parts of absolute space to be at mutual rest - otherwise, with respect to what part is absolute motion to be taken? In fact, Newton held the stronger view that the parts were necessarily motionless, and in two well-known passages argued for this claim: first in the unpublished essay De Gravitatione, and again in the Scholium to the Definitions in his Principia. The arguments have been rather widely discussed, to various ends, but, I will argue, they have been misunderstood in a number of ways.

More specifically, in $\S 1$ I will argue (against Nerlich, 2005, for instance) that De Gravitatione contains two arguments for immobility, while the Scholium contains only one of them. In particular, the 'identity argument' that is the focus of recent discussion (and this paper) is only found in De Gravitatione. This point is not made merely to correct a mistake in the literature, but to show that the premises of Newton's argument do not appear in his more considered, published works, and thus do not have the same status as his core views.

$\S 2$ will canvas some possible interpretations of the identity argument and their problems, and explicate the most charitable, natural reading - according to which the premises are consistent with absolute space as conceived by Newton. One reason that the argument has attracted attention (e.g., DiSalle 1994) is that it also seems to show that Newton denied a 'substantival' view of space: its premises imply rather immediately that worlds that (putatively) differ only in the absolute location of the material universe do not in 
fact differ at all. Unfortunately for this interpretation of Newton, we shall see in $\S 3$ that the premises also entail that all motion is the relative motion of bodies. The denial of this doctrine is of course essential to Newton's natural philosophy. Since the problematic argument appears only in his unpublished writings, it thus seems best to bracket it from his considered views - as a result, the identity argument is, after all, no evidence that Newton seriously or consciously denied substantivalism.

\section{The Arguments}

In the first part of De Gravitatione, Newton gives a detailed criticism of the conception of space found in Descartes' Principles of Philosophy, according to which space and matter are identified and hence the parts of space are in motion. Then he turns to a positive account of the nature of space as he conceives it. Numbered point 3 is the assertion that 'the parts of space are motionless'. For our purposes, the relevant argument for this claim is as follows:

[(i)] If they moved, it would have to be said ... that it is translation out of space into space, that is out of itself, unless perhaps it is said that two spaces everywhere coincide, a moving one and a motionless one. [(ii)] Moreover, ... the parts of space are individuated by their positions, so that if any two could change their positions, they would change their individuality at the same time and each would be converted numerically into the other. The parts of duration and space are understood to be the same as they really are only because of their mutual order and position; nor do they have any principle of individuation apart from that order and position, which consequently cannot be altered. (2004, 25)

Paragraph 6 of Newton's Scholium ${ }^{1}$ makes the same claim - again obviously opposing Descartes - and offers the following argument.

Just as the order of the parts of time is unchangeable, so, too, is the order of the parts of space. Let the parts of space move from their places, and they will move (so to speak) from themselves. For times and spaces are, as it were, the places of themselves and of all things. All things are placed in time with reference to order of succession and in space with reference to order of position. It is of the essence of spaces to be places, and for primary places to move is absurd. They are therefore absolute places, and it is only changes of position from these places that are absolute motions. (1999, 410)

\footnotetext{
${ }^{1}$ In fact the seventh paragraph - but the paragraph that would have been numbered ' 6 ' by Newton if he had carried on numbering them beyond ' 4 '.
} 
The purpose of this section is to clarify the relationship between these two passages, and in particular to show first that De Gravitatione contains two arguments, which I have numbered (i) and (ii); and second that (ii) does not appear in the Scholium, rather the argument is along the lines of $(i)$. In the first place, the point is important to make because it is not always understood: for instance, Nerlich (2005) does not really recognise (i) as a distinct argument and so mistakenly takes Newton's argument in the Scholium to be the same as (ii) (indeed his paper is an analysis of (ii), but is subtitled 'On Paragraph Six of Newton's Scholium'). But of considerably greater importance for the evaluation of (ii) is the question of whether it appears in Newton's published works - such as the Scholium, but not De Gravitatione - and hence how central it is to Newton's views.

Certainly the two passages are related; in the first place they are both clearly concerned with distinguishing Newton's view of space from Descartes'. However, since Paragraph 6 (like the rest of the Scholium) says nothing explicit about the identity criteria of the parts of space, it is prima facie plausible that (ii) does not appear. To confirm this suspicion, we need to analyse the arguments in more detail, particularly to see how Paragraph 6 of the Scholium corresponds without remainder to (i) of De Gravitatione.

(i) This argument ${ }^{2}$ assumes that space is the ultimate standard of rest. That is, earlier in De Gravitatione Newton argues at length that 'true' and 'philosophical' motion cannot be defined in terms of relative motions - even motions relative to contiguous surroundings, as Descartes proposed. For instance, a body's contiguous surroundings will surely disperse over time, since matter is in constant flux: but if the original place of a body no longer exists, it is impossible to say how far it has travelled from that place, and consequently what its relative speed was. That is, 'not even God himself could define the past position of any moving body .... and since velocity depends upon the length of the space passed over in a given time, it follows that the moving body has no velocity ...' $(2004,20)$ Newton proposes instead that motions can be referred to an independent reference frame, space; 'it is necessary that the definition of ... local motion be referred to some motionless being such as extension alone or space in so far as it is seen to be truly distinct from bodies.' (2004, 20-1) Obviously it is not sufficient for Newton's space to be independent of bodies, it must also be static, else the same problem will arise for his account of motion - absolute places can disperse.

Newton thinks that he can secure this result from the assumption that motion is ultimately motion with respect to space. For what would it be for a part of space to move? Not to move relative to bodies apparently. Newton doesn't say why explicitly, but if space were in motion merely because bodies move relative to it, then its motion would be symmetrical with that of bodies, and its status as an ultimate standard of rest for bodies is undermined. Thus if a part of space moves it moves with respect to space: i.e., truly.

\footnotetext{
${ }^{2}$ Thanks to Oliver Pooley for a number of helpful comments about my reading of (i).
} 
But for Newton, true motion is motion out of one part of space and into another. That is, 'locomotion' is traditionally understood as 'change of place', a view endorsed by Newton both here (Definition 4, 2004, 13) and in the Scholium: 'absolute motion is the change of position of one body from one absolute place to another' $(1999,409)$. But according to Newton, places are literally the parts of space, the points, lines, surfaces and solids that are found everywhere $(2004,13 ; 1999,409)$. Hence a part of space could only move truly in one of two ways: either by moving 'out of itself' in some hard-to-imagine sense, or by moving out of a part of a second space. Obviously Newton considers both of these proposals as risible; the argument is a reductio.

(It is not clear how this argument secures Newton against the motion of the parts of space relative to one another. Why should we not define another sense of the motion of places, not as motion out of place, but a change of distance to other places. I don't see how Newton's argument rules out motion of this kind. But of course it is the relative motions of the parts of space that Newton identifies above as making a definition of velocity impossible for Descartes. Looking ahead a little, since the Scholium contains only (i) it does not after all secure space against the problematic kind of motion; immobility apparently must be stipulated, not demonstrated from more fundamental principles. ${ }^{3}$ )

(ii) 'Moreover' clearly indicates the start of a second argument; one that in no obvious way even shares premises with the first. It reasons from the claim that the identities of the parts of space arise entirely from their distance (or 'metrical') relations to other parts of space: from 'their position'. Then, for instance, if parts $p$ and $q$ were, putatively, to swap positions, then $p$ would come to posses all the metrical relations that $q$ had earlier, and vice versa, and so $p$ would become $q$, and vice versa. But that is to say that $p$ and $q$ would end up where they started, and that no parts of space would have moved after all.

It's worth emphasising that Newton does mean metrical relations, not monadic positional properties, particular to each part, on which the relations supervene. (One might imagine such properties receiving co-ordinates, and then the supervenient metrical relations being determined by the application of the Euclidean metric to the co-ordinates.) That is, Newton's 'absolutism' is not here opposed to 'relationism' in the sense of irreducible relations between the parts of space. For he quite clearly refers to the 'mutual' positions of the parts of space ('positiones inter se partes' in the original Latin). Moreover, there is no reason to think that Newton denies such relationism anywhere: there is, as far as I am aware, no passage in which he denies primitive spatial relations. ${ }^{4}$

\footnotetext{
${ }^{3}$ Looking ahead to the end of the paper, although (ii) does demonstrate the relative immobility of the parts of space, since Newton cannot consistently hold it, he has no demonstration at all.

${ }^{4}$ Thanks to Alyssa Ney for pressing me on this issue.
} 
The 'identity argument' - (ii) - is interesting and sophisticated, and ultimately incompatible with Newton's overall views, as we shall see when we unpack it in detail in the following sections. For now, the point is that we are dealing with a completely separate line of thought from (i). Moreover, it is only the first argument that is to be found in the passage quoted from the Scholium, as we can see by reading it sentence-by-sentence.

First we have that the parts of space are motionless. Next we are told now that a place can only move by moving out of itself; the possibility of 'two spaces' is not raised here. In De Gravitatione we justified this assertion on the basis of two claims: first that any motion of the parts is to be referred only to space, not to bodies, and second that motion relative to space means specifically motion out of a place. The latter claim is made explicitly by Newton in the Scholium as well as De Gravitatione. The former was not made explicitly in De Gravitatione, but the next two (and a half) sentences from the quoted passage give a justification. That is, they say that the parts of space are the ultimate places and as such are the places of themselves - there is nothing more fundamental which could be their places. Then Newton points out that for 'primary places' to move would be absurd - at least because if they moved it would be 'from themselves'.

Finally, Newton concludes that absolute motions are changes of absolute positions. It is not entirely clear whether this conclusion is supposed to follow from the immobility of places or from the fact that the parts of space are 'primary' places; I am inclined to say the latter (following Nerlich, 2005, §2.1). (Since Newton has already defined absolute motion to be change of absolute place, it may seem unclear why he needs an argument here. I agree with Nerlich that what he means to show is that motion in the 'true' or 'philosophical' sense is absolute motion, as defined. I also agree that Newton's argument is a priori and metaphysical.)

This analysis exhausts the passage quoted from the Scholium, leaving no part that could contain (ii). The only words that are even remotely reminiscent are that things are placed 'in space with reference to order of position', which perhaps reminds one of the claim that the parts of duration and space are 'understood to be the same as they really are only because of their mutual order and position'. But the Scholium makes no claims at all about the individuation of the parts; there is, for instance, no analogue of 'nor do they have any principle of individuation apart from that order and position'. Instead, the reference to 'order' is surely to say that all things (both bodies and places) have their metrical relations in virtue of the metrical relations of the parts of space. For instance, the Earth and the Sun are (approximately) $10^{11} \mathrm{~m}$ apart, in virtue of occupying points of space that are $10^{11} \mathrm{~m}$ apart.

The discussion of the immobility arguments may seem unnecessarily drawn-out; perhaps when pointed out, it's rather obvious that the Scholium does not invoke the identity argument. But it was worth being careful. Most importantly, when we see that the identity argument is fallacious, the fact that it only appears in a speculative unpublished essay means that we are justified in excluding it from Newton's core views on space. 


\section{The Identity Argument}

From now on our attention is focussed on De Gravitatione, and specifically to (ii), the identity argument. In this section we will consider three main readings, first one recently proposed by Nerlich and then two others. Nerlich's reading commits Newton to an obvious contradiction (as Nerlich points out), while the other two are more charitable. However, only the last fits well with what Newton says, though it casts his argument into terms of contemporary modal semantics.

\subsection{Identity of Indiscernibles for Individuals}

Nerlich takes the 'most obvious interpretation' $(2005,122)$ of the identity argument to involve an appeal to the Principle of the Identity of Indiscernibles (PII). He doesn't say explicitly how he understands the principle here (below we shall see some alternatives), but it seems clear that he takes it to mean that if any two parts of space are indistinguishable with respect to their metrical relations to the other parts of space, then they are strictly identical. (From now on let us generally refer to points of space for simplicity, though the discussion applies equally to any of the parts of space.)

Let us call an instantaneous specification of the metrical relations of the points of space the state of space. Then Newton's argument for the immobility of space supposedly proceeds as follows (expanding a little on Nerlich). Consider any two states, $s_{1}$ and $s_{2}$, such that in $s_{2}$ a point $q$ has all the metrical properties that a point $p$ has in $s_{1}$. Then by PII, $q=p$. Thus in an evolution from $s_{1}$ to $s_{2}, q$ cannot have taken the place of $p$ : instead of moving, $q$ 'would be converted numerically into' $p$. And similarly for all points and parts of space. $Q E D$.

This reading fits with what Newton says, but it has, as Nerlich points out, a notable defect. As we saw, Newton claims that points have no "principle of individuation apart from [their] position': from their metrical relations to one another. Thus it seems that a point cannot be picked out in any way other than in terms of its metrical relations to other points. But the same applies to the other points - thus there are no antecedently identified points relative to which others can be identified. That is to say, the metrical properties of points are purely qualitative, not relations to particular identified or named points. ${ }^{5}$

But the symmetries of Euclidean space mean that every point has the very same purely qualitative metrical relations as every other; pick any set of points with whatever mutual relations to one another and to $p$, and there is set a set of points with the same mutual

\footnotetext{
${ }^{5}$ One might try to challenge this claim by pointing out that the points of space bear metrical relations to bodies as well as points. I can, for instance, describe the various relations that places bear to the tip of my finger: one is coincident with it, others are $1 \mathrm{~m}, 1 \mathrm{~km}$ or 1 parsec away. This proposal won't save Newton from the looming problem, for he believed that space existed before matter $(2004,26-7)$, and so at times at which points were not distinguishable by their metrical relations to matter. The contradiction described just below would hold at least at such times, and that is obviously bad enough.
} 
relations and relations to $q$. There is no way to distinguish points of Euclidean space by their purely qualitative relations to other points.

Thus if Newton intends that the metrical relations between points are purely qualitative, and if he employs PII as suggested, then he is committed, by another application of PII, to the conclusion that all the points of space are strictly identical - in contradiction with the standard model of Euclidean geometry assumed by his physics. Either Newton is badly confused or there is something wrong with the interpretation; I want to explain what seems to me a more natural reading - one which avoids this problem.

\subsection{Identity of Indiscernibles for Worlds and States}

Nerlich mentions Leibniz's use of PII in the Correspondence with Clarke, to remark on its apparent similarity to his account of Newton's argument. However, he does not note the important fact that Leibniz invokes PII in several distinct senses. First Leibniz employs PII for individuals in a single world: for instance, there can be no atoms because they are supposed to be indistinguishable individuals $(1956,36)$. But he also takes it to apply to worlds: for instance, because of PII it is not possible that 'the universe could have had at first another position of time and place, than that it actually had; and yet that all the parts of the universe should have had the same situation among themselves, as that they actually had.' $(1956,37)$ The worlds are indistinguishable, and hence identical.

Leibniz held both forms of PII, but there is no obvious reason that one should. Newton could, for instance, deny PII for individuals in a world but uphold it for worlds. That is, he could avoid the problem described by Nerlich by denying that the indistinguishability of points by their metrical relations implies their strict identity; he could indeed naturally claim that they are diverse in virtue of bearing non-zero distance relations to one another, like Black's spheres (1952). ${ }^{6}$ Then Newton could argue as follows. Suppose that there were two worlds $w_{1}$ and $w_{2}$ in which space is Euclidean and such that in $w_{2}$ points $p$ and $q$, respectively, have all the metrical properties that $q$ and $p$, respectively, have in $w_{1}$. Since the worlds are Euclidean, points bear just the same non-qualitative metrical relations to one another in both worlds, and since these are the only properties by which the worlds might be distinguished, $w_{1}$ and $w_{2}$ are indistinguishable. ${ }^{7}$ By PII, $w_{1}=w_{2}$, and so $w_{2}$ cannot be viewed as a world in which $p$ and $q$ have changed positions from $w_{1}$; but no change of position means no motion. And similarly for all points and parts of space. $Q E D$.

Thus if Newton argued this way - using PII for worlds not individuals - he would have avoided Nerlich's problem. There are, however, two problems with this reading. The first is easy to fix. Newton's argument concerns motion, and so change of position over time in

\footnotetext{
${ }^{6}$ Actually that won't suffice; a single sphere in a cylindrical space will also be at a non-zero distance from itself. It would be better to claim that a point is distinct from any other with which it is not coincident, regardless of the other relations it bears to itself.

${ }^{7}$ For simplicity, we suppose that these worlds contain no bodies, though the argument can still be made as long as the bodies have the same relations in the two worlds. What we want is to ignore the relations of points to bodies. See also footnote 5 .
} 
a single world, not difference of position in different worlds. Thus an argument like this will have to invoke PII, not for worlds, but for states. These principles are not automatically equivalent: for instance, one might hold that duplicate worlds exist, even though these could be identical temporal sequences of strictly identical states. ${ }^{8}$

But the reading is easily fixed: just replace 'world' with 'state' and ' $w_{i}$ ' $(i=1,2)$ with ' $s_{i}$ ' throughout. It is, in this context, interesting to note that Leibniz also held PII for states, and used it to conclude that systems could not differ only in their overall absolute velocity: 'two states indiscernible from each other, are the same state' $(1956,38)$.

The fix is easy and unproblematic, but the switch is worth remarking. As we go on we will be using the concepts of possible worlds semantics, but we will be applying them to instantaneous states, not full diachronic worlds. We have to, because, as we just noted, the question is whether points can move in a world; whether there are states in which points have different locations. And the strategy is possible because the possible states of a system have a structure just like the universe of possible worlds; of course, states are just like worlds that last for only an instant.

The second problem is more serious. In the reading under discussion, all the work is done by the fact that space is Euclidean in the two worlds, and hence that the same qualitative metrical relations are instantiated in both; that $q$ 'would be converted numerically into' $p$ (and vice versa) seems to play no role. That is, the problem with this reading is that it does not give a proper role to, or interpretation of, Newton's claim that points are individuated by their metrical relations - a point I had not fully appreciated until I read Nerlich's essay. ${ }^{9}$ PII between worlds or states will not do justice to Newton and we need to try again.

\subsection{Representation $\mathrm{De} R e$}

One suggestion is that Newton rejects PII for individuals in a single world (or rather state), but maintains it for individuals in different worlds (or states). Then his argument for the immobility of space would say that if there were a state $s_{2}$ in which a point $q$ had all the metrical properties that $p$ has in a state $s_{1}$, then by PII, $p=q-q$ would be 'converted numerically' into $p$. But then neither $q$ nor, mutatis mutandis, any other part of space could change place. Moreover he could again dodge Nerlich's problem by denying that PII applied directly to the points of space in any one state. But that wouldn't get Newton far. For since all the points of space in $s_{2}$, including $q$, have the same qualitative metrical relations as each other, they are all indistinguishable from $p$. Thus by PII for individuals

\footnotetext{
${ }^{8}$ In this case one could capture the much of the intent of PII with the weaker principle that any worlds which agree on the purely qualitative metrical relations between points do not differ in any non-qualitative metrical relations between points. That is, two worlds that are exactly alike in qualitative metrical relations may not be the very same world (even if they, say contain no bodies), but they do not differ in the relations between any identifiable points.

${ }^{9}$ Newton's views on individuation are not entirely absent from this argument. They are needed to conclude that indistinguishability is indistinguishability with respect to purely qualitative relations.
} 
in different states, $p$ is identical to all of them, and by the transitivity of identity, they are again all one, contrary to Newton's understanding of Euclidean space.

The problem here is with Newton's assertion of the numerical identity of the points in different states. But perhaps he really has something weaker in mind than strict identity here. Consider the similar case in possible world semantics; modal claims about someone in the actual world generally depend on what that person gets up to in other possible worlds - on some kind of 'identification' of individuals across worlds. One naturally first thinks of this identification as a matter of strict identity, but that view can lead to problems one at least has to deal with the fact that an individual will have inconsistent properties in different worlds. It's no business of this paper to argue for (or against) any account of such things here, but only to point out that there are alternative proposals to strict identity.

The general notion here is what Lewis (1986, Chapter 4) calls 'representation de re'. Facts about what an individual would or could or could not or must do depend on what the individual that represents him de re does in other worlds. For instance, is it true that if Bush had not won in 2000 he would have had a successful career in oil? We could say 'yes' if in the nearest worlds in which the person representing Bush de re lost the election, they went on to be big in oil; otherwise no. According to one account, the individual that represents him de re is strictly identical to him, but in other accounts that need not be the case; modal facts about him depend not on what that very individual does in other possible worlds, but on what some (generally) non-identical individual does in them.

Lewis' own proposal is that representation de re is determined qualitatively: specifically, an individual $x$ is represented de re in another possible world by the individual in that world who is most qualitatively similar to $x-x$ 's 'counterpart'. Bush's counterpart is the individual in a world who most closely resembles our Bush, and it is that individual's career that concerns us for the evaluation of modal propositions.

A third kind of account holds that representation de re is not determined either by the relation of strict identity or by the counterpart relation, but by some 'non-qualitative property' of 'thisness'. According to such an account it is in principle possible that the closest possible world in which Bush lost is qualitatively just like ours, except that nonqualitative properties pick out a person just like our Gore as representing Bush de re (and vice versa); if so, Bush would have warned of global warming instead of working in oil if he had lost.

Other accounts of representation can be conceived, and debated, but for our purposes two points are relevant. First, representation de re does not necessarily mean strict identity. Second, without the terminology of 'representation' it is not unreasonable to speak of 'identity' since the issue is one of 'identification' across worlds - and even to speak of 'numerical identity', to distinguish the issue from mere qualitative identity or indistinguishability. If we read Newton's claims about the individuation in this way then we can see that he is in fact proposing a principle of representation de re for the parts of space. Specifically, he is effectively claiming that de re representation of points is determined by their qualitative metrical relations (for brevity we will generally take the modifier de re 
as read in all that follows). Such a view suggests a Lewis-style counterpart account of representation: $p$ in $s_{1}$ is represented by the point in $s_{2}$ which has the most similar metrical properties. ${ }^{10}$ But Newton is not so specific, and counterpart theory is surely not the only account of representation compatible with the principle - qualitative properties can in principle be compared in other ways than maximal similarity.

In more detail, let us postulate that only a lacuna in Newton's knowledge of modal semantics led him to phrase the argument as he did, and so rephrase everything in terms of representation rather than numerical identity. First we reformulate Newton's claim about identity, that the parts of space are 'the same as they really are only because of their ...position; nor do they have any principle of individuation apart from that ... position' (i.e. metrical relations to one another). Read in terms of strict identity, this claim says that if two points have the same metrical relations to other points in two states then they are the same point. But that rendering leads to the catastrophic conclusion that Euclidean space contains a singe point. In terms of representation de re, 'Newton's principle' is that the representation of points supervenes on the metrical relations of points to one another. In particular, if two points in different worlds or states have the same metrical relations to other points then they represent the same points.

Now we can interpret Newton's argument (ii) as follows. Suppose that any set of points in $s_{1}$ not including $p$ or $q$ is represented in $s_{2}$ as bearing the same metrical relations as they do in $s_{1}$ : Newton's argument only considers whether $p$ and $q$ can move, so we leave the rest of space unmoved by assumption. ${ }^{11}$ Suppose further that any set of points in $s_{1}$ not including $p$ or $q$ is represented in $s_{2}$ as bearing the same relations to $q$ as they do to $p$ in $s_{1}$. That is, we use the representation relation between points to understand how $p$ and $q$ 's places in $s_{1}$ are represented in $s_{2}$, and then suppose $p$ and $q$ are swapped with respect to those places.

By supposition, $p$ in $s_{1}$ and $q$ in $s_{2}$ have the same metrical relations to other points and so, by Newton's principle, represent the same point, namely $p$ (an individual can represent itself of course). Which is to say that in any state in which $q$ took $p$ 's place, $q$ would represent $p$, and hence represent $p$ as being just where it always was; thus it is impossible for $p$ to move. And similarly for the other parts of space. $Q E D$.

(More generally, Newton's principle means that the metrical relations of any of the parts of space 'cannot be altered', assuming, as he did, that the geometry of space - i.e., the metrical relations between its parts - remains unchanged. For the only way that a part

\footnotetext{
${ }^{10}$ In which case, as Pooley pointed out to me, the kind of view that I am attributing to Newton is a relative of Sider's $(2001, \S 5.8)$ temporal counterpart theory.

${ }^{11}$ It is of course hard to see how, given the homogeneity of space and Newton's principle, how any determinate representation relation can hold between the 'unmoved' points. Still, Newton's argument does seem to assume that space is fixed apart from the putatively permuted parts. I take it that the assumption is a concession to his imagined opponent and so, for the purposes of the argument, quite justified. On the other hand, the absence of any determinate relation will be used in the next section to undermine Newton's commitment to the principle. (Thanks to Colin Klein, inter alia, for pointing out the need for clarification here.)
} 
can move is if there are two states that differ in how they represent the metrical relations of the part. But if space has the same geometry in two states, then by Newton's principle they cannot represent anything differently about the metrical relations of parts: supervenience means no difference in representation without a difference in metrical relations.)

The formulation of Newton's argument in terms of representation completely avoids the problem raised by Nerlich. The only objection might be to the very introduction of representation de re; doesn't it make the formulation a matter of rational reconstruction rather than interpretation? I would agree in the limited sense that it introduces logical resources not available to Newton, much as we cast his views on kinematics in terms of spacetimes. But on the other hand it seems to me clearly to be a sympathetic reworking that captures Newton's intentions while saving him from a pretty glaring error. Indeed, surely he would have noticed Nerlich's problem easily, so it seems more plausible and charitable to say that he was was reaching for something like representation, rather than that he made such a poor argument. Providing that we explicitly acknowledge that we are adding a small sophistication to Newton, it is fair enough to claim that we have an interpretation that is faithful to his intentions in De Gravitatione.

That said, in the next section we will see why this argument is incompatible with Newton's core doctrines about space and motion (in short it suffices to show that all motion is the relative motion of bodies). Thus I will propose that Newton's identity argument is not itself important to Newton's fundamental views.

First I would like to make one further remark about Newton's claim that because the identity (or representation de re) of a point is determined by its metrical properties, those properties 'consequently cannot be altered'. Maudlin (1990) has reasonably read this as a statement of 'metric essentialism': the view that the parts of space hold their metrical properties essentially. However, Newton's claim about identity, as I have formulated it in terms of representation de re, does not imply such essentialism, which one might take as an objection to my reading - viz. that it leaves something out of Newton's views.

For the following is perfectly compatible with Newton's principle as I (re)formulated it: let space in state $s_{1}$ be everywhere Euclidean except for four regions of non-Euclidean geometry so arranged that there is one to which any other two are at right angles. That one can be taken as the 'origin' of a some natural axes, while the others pick out the directions of the axes. And let space in $s_{2}$ be the same except for a fifth non-Euclidean region far distant from the others. As we noted, a counterpart view of representation is consistent with Newton's principle, so it is consistent with Newton's views that a point $p$ in $s_{1}$ is represented in $s_{2}$ by that point which bears the same relations to the four axis-defining regions in $s_{2}$ as $p$ bears to the regions in $s_{1}$ - its 'metrical counterpart'. But this point will not have all the same metrical properties as $p$, since it bears relations to the fifth non-Euclidean region, which $p$ does not, since in $s_{1}$ the region is absent. Thus $s_{1}$ and $s_{2}$ represent $p$ as having different metrical properties, contrary to metric essentialism - but not contrary to Newton's principle.

Of course what's most notable about the failure of metric essentialism here is that it 
requires space to change its metric. (Not all failures are of this kind: if points in regions of different geometry were permuted they would change their metrical properties, but the geometry of space would stay the same - this situation is ruled out by Newton's principle of course.) In the context in which someone takes space to necessarily have Euclidean geometry, my reading of Newton does imply that he is a metric essentialist. Moreover, it is not at all clear what Newton would have said about non-Euclidean geometries; would points still have possessed their metrical properties necessarily, or would he have countenanced changes of geometry? It's impossible to say, but at least as likely that he would as that he wouldn't. I don't see any problem here for my reading.

\section{Kinematic Relationism}

One interesting consequence of Newton's claims about the identity of points in De Gravitatione is that the kind of 'shift' proposed by Leibniz above does not lead to a distinct world. That is, suppose that two worlds differ only in the position of the material universe in absolute space. Since space is Euclidean (and hence metrically homogeneous) every point in one world is metrically indistinguishable from every point in the other. Hence no particular point in one world can be unequivocally identified as representing any particular point in the other; if any represents $p$ then they all do, since representation supervenes on the metrical relations of points to one another. So no points are represented as standing in different relations to matter in the two worlds.

DiSalle $(1994,267)$ takes this point as showing that Newton is not, in the contemporary jargon, a 'substantivalist' about space. According to (most versions of) this view, the absoluteness of absolute space can be captured by saying that shifts of the kind suggested by Leibniz produce distinct worlds - contrary to Newton's principle, as we have just seen. ${ }^{12}$

Following Pooley $(2002,43)$ I want to make one caution about substantivalism before arguing that Newton's principle - hence Newton's alleged anti-substantivalism - is largely irrelevant to understanding Newton's overall views. For it is not in fact clear that the possibility of shifts in absolute space implies the proliferation of worlds that Leibniz found so objectionable. Lewis (1986, 230-35) discusses the possibility that an individual might have multiple counterparts in a single world, in which case one world would correspond to multiple possibilities. In the present case, every other point potentially represents a different possibility for a point. But (assuming an asymmetric distribution of matter) each other point stands in different relations to matter, and so potentially represents a different possible absolute position for matter - all within a single world. Thus it is not unproblematically the case that a 'Leibniz shift' produces a world that represents the location of matter differently.

\footnotetext{
${ }^{12}$ It is not at all clear that Newton's principle is incompatible with substantivalism in the sense introduced in 1922 by Johnson $(1964,165-6)$ : that bodies can bear relations to points as well as each other.
} 
Let us put this caution aside, and say that in some senses at least Newton's views on the identity of points are contrary to substantivalism.

This is a very surprising result, because substantivalism seems such a natural way of cashing out one sense of the 'absoluteness' of absolute space, its independence from matter. (Not to mention of course Clarke's acceptance of the reality of shifts.) However, we can quickly see that Newton's principle - and hence the inference to anti-substantivalism cannot be accommodated in his central views.

The problem is that the reasoning which (perhaps) enabled Newton to avoid Leibniz's shift, shows also that bodies cannot stand in different relations to space at different times: it immediately follows that any changes are only in the relations between bodies, contrary to Newton's clear view that motion is not merely relative. For consider two states in which the geometry is Euclidean (though again homogeneity suffices), so that every point in one world is metrically indistinguishable from every point in the other. Since representation de re supervenes on the metrical relations between points, one state cannot unequivocally represent any point as standing in any different relation to bodies from the other. But bodies can only move with respect to space - i.e., absolutely - if they can occupy different parts of space at different times; and that is impossible if matter isn't represented as occupying different parts of space in the possible states of the world.

Put most simply, if points are to be 'reidentified' by their qualitative metrical relations to one another, then there is no basis to 'identify' a point $p$ with any particular point at a later time: and thus 'not even God himself could define the past position of any moving body' ${ }^{13}$ Of course this is not a conclusion that Newton could accept as part of his general view: a main point of the Scholium is to show that 'absolute and relative rest and motion are distinguished from each other' $(1999,411)$.

This conclusion is fairly obvious, but not as obvious as the consequences of Nerlich's reading; it could be overlooked in drafting a small part of a complex essay such as De Gravitatione. It is interesting that the identity argument did not appear in the Scholium even though argument (i) did. Though I have no evidence, one might speculate that Newton realised his error when he came to prepare his work more carefully for publication, and even rejected the argument. At any rate, the fact that Newton's views on identity do not appear in his published works (as we carefully established in §1) coupled with the fact that they are incompatible with his core, published doctrines, means that we do best not

\footnotetext{
${ }^{13}$ Two remarks: first, it does no good to postulate some additional criterion of temporal 'identity', independent of metrical relations. That would undermine the identity argument, for then $q$ could take $p$ 's place while remaining $q$ according to the new criterion. The crux is that states are (possible) states of the world at different times, so the account of representation across states is the account of temporal identity.

Second, the argument given in the main text would not be undermined in the way that the argument against substantivalism might be. One might try to say that a later state represents many possible motions, corresponding to different representations of the points, and hence of the location of matter relative to space. But Newton's doctrine is not merely that matter can move with respect to space, but that it has a determinate motion with respect to space - and that requires a series of states with bodies in determinately different positions.
} 
to take them seriously as part of Newton's overall position. And that is to say that we should not take Newton to deny substantivalism after all.

\section{Conclusion}

This essay aimed to clarify Newton's views on the immobility of space, particularly those concerning the individuation of points: what does it mean to say that points are identified by their metrical relations to one another? If it meant some version of PII, then his views rather obviously imply the absurd conclusion that all points of space are one. It seems much more reasonable to think that Newton means instead that metrical properties determine the de re representation of points in different worlds and states. This view however implies that all motion is the relative motion of bodies, contrary to Newton's key insight in kinematics. Thus we should conclude that the identity argument for immobility is peripheral to Newton's views: in particular, we should not take seriously the suggestion that he was opposed to substantivalism.

\section{Bibliography}

Black, M., 1952, "The Identity of Indiscernibles", Mind, 61: 153-164.

DiSalle, R., 1994, "On Dynamics, Indiscernibility, and Spacetime Ontology", British Journal for the Philosophy of Science, 45: 265-287.

Johnson, W. E., 1964, Logic, New York, Dover Publications.

Leibniz, G. W., S. Clarke and I. Newton, 1956, The Leibniz-Clarke Correspondence, Together with Extracts from Newton's "Principia" and "Opticks", H. G. Alexander (ed.), Manchester: Manchester University Press.

Lewis, D. K., 2000, On the Plurality of Worlds, Blackwell Publishers Malden, Mass.

Maudlin, T., 1990, "Substances and Space-Time: What Aristotle would have Said to Einstein", Studies in History and Philosophy of Science, 531-561.

Nerlich, G., 05, "Can Parts of Space Move? on Paragraph Six of Newton's Scholium", Erkenntnis, 62: 119-135.

Newton, I., 1962, Unpublished Scientific Papers of Isaac Newton; a Selection from the Portsmouth Collection in the University Library, Cambridge, A. R. Hall and M. B. Hall 
(eds.), A. R. Hall and M. B. Hall (trans.), Cambridge, UK: Cambridge University Press.

Newton, I., 1999, The Principia: Mathematical Principles of Natural Philosophy, I. B. Cohen and A. M. Whitman (trans.), Berkeley: University of California Press.

Newton, I., 2004, Newton: Philosophical Writings, A. Janiak (ed.), Cambridge: Cambridge University Press.

Pooley, O., 2002, The Reality of Spacetime, D.Phil thesis, Oxford University.

Sider, T., 2001, Four Dimensionalism: An Ontology of Persistence and Time, Oxford, Oxford University Press. 